\title{
Gray Matter Variation in the Posterior Superior Temporal Gyrus Is Associated with Polymorphisms in the KIAA0319 Gene in Chimpanzees (Pan troglodytes)
}

\author{
William D. Hopkins, ${ }^{1}$ Nicky Staes, ${ }^{2,3,4}$ @Michele M. Mulholland, ${ }^{1}$ Steven J. Schapiro, ${ }^{1,5}$ \\ Madeleine Rosenstein, ${ }^{2}$ Cheryl Stimpson, ${ }^{6,7}$ Brenda J. Bradley, ${ }^{2}$ and Chet C. Sherwood ${ }^{2}$
}

https://doi.org/10.1523/ENEURO.0169-21.2021

${ }^{1}$ Department of Comparative Medicine, Michale E Keeling Center for Comparative Medicine and Research, The University of Texas MD Anderson Cancer Center, Bastrop, TX 78602, ${ }^{2}$ Department of Anthropology and Center for the Advanced Study of Human Paleobiology, The George Washington University, Washington, DC 20052, ${ }^{3}$ Behavioural Ecology and Ecophysiology Group, Department of Biology, University of Antwerp, Wilrijk, Belgium $2000,{ }^{4}$ Centre for Research and Conservation, Royal Zoological Society of Antwerp, Antwerp, Belgium 2000, ${ }^{5}$ Department of Experimental Medicine, University of Copenhagen, Copenhagen, Denmark 1017, ${ }^{6}$ Department of Pathology, Uniformed Services University, Bethesda, MD 20817, and ${ }^{7}$ Henry M. Jackson Foundation for the Advancement of Military Medicine, Bethesda, MD 20817

\begin{abstract}
Determining the impact that the KIAA0319 gene has on primate brain morphology can provide insight into the evolution of human cognition and language systems. Here, we tested whether polymorphisms in KIAA0319 in chimpanzees account for gray matter volumetric variation in brain regions implicated in language and communication (particularly within the posterior superior temporal gyrus and inferior frontal gyrus). First, we identified the nature and frequencies of single nucleotide variants (SNVs) in KIAA0319 in a sample of unrelated chimpanzees (Pan troglodytes spp.). Next, we genotyped a subset of SNVs (those important for gene regulation or likely to alter protein structure/function) in a sample of chimpanzees for which in vivo T1-structural magnetic resonance imaging scans had been obtained. We then used source-based morphometry (SBM) to test for whole-brain gray matter covariation differences between chimpanzees with different KIAA0319 alleles. Finally, using histologic sections of 15 postmortem chimpanzee brains, we analyzed microstructural variation related to KIAA0319 polymorphisms in the posterior superior temporal cortex. We found that the SNVs were associated with variation in gray matter within several brain regions, including the posterior superior temporal gyrus (a region associated with language comprehension and production in humans). The microstructure analysis further revealed hemispheric differences in neuropil fraction, indicating that KIAA0319 expression may be
\end{abstract}

\section{Significance Statement}

Studying the impact of language-related genes on primate brain morphology can provide insight into the evolution of human cognition and language systems. Here, we show that two KIAA0319 variants in chimpanzees are linked to differences in gray matter volume of the posterior superior temporal gyrus, a region associated with language comprehension and production in humans. Examination of the microstructure of this region showed that one KIAA0319 variant is associated with hemispheric differences in neuropil fraction, indicating that KIAA0319 expression may be involved in the formation and maintenance of synapses, dendrites, or axons within a region directly involved in communication. Together, these findings suggest that KIAA0319 variants may underlie individual variation in auditory processing and associated brain regions in nonhuman primates. 
involved in regulation of processes related to the formation and maintenance of synapses, dendrites, or axons within regions associated with communication.

\section{Key words: chimpanzees; communication; gray matter; KIAA0319; language; neuropil fraction}

\section{Introduction}

Characterizing the behavioral, neural and genomic systems that distinguish humans from other primates has been a central focus of comparative research. In humans, there are several key connected brain regions implicated in the production and comprehension of language, particularly within the left hemisphere, including the basal ganglia, supplemental motor, inferior frontal, and posterior superior temporal gyri (Goulven and Tzourio-mazoyer, 2004; Friederici, 2015; Skeide et al., 2016). Further, studies have discovered several genes that account for individual and phylogenetic variation in linguistic functions and their underlying neural systems (Fisher and Marcus, 2006; Fisher and Scharff, 2009; Preuss, 2012; Pfenning et al., 2014). Arguably, the most well-known so-called language gene is FOXP2 (Lai et al., 2001; Enard et al., 2002). Individuals with a FOXP2 R553H missense mutation exhibit oro-facial dyspraxia and concomitant decrements in gray matter volume in the basal ganglia, supplemental motor area, anterior cingulate, and inferior frontal gyrus (Vargha-Khadem et al., 2005).

The gene KIAA0319 has similarly been implicated in language functions of humans. KIAA0319 polymorphisms have been repeatedly associated with developmental dyslexia, a reading disorder marked by difficulties learning associations between sounds with letters and/or written words (Cope et al., 2005; Dennis et al., 2009; Scerri et al., 2011; Luciano et al., 2013; Sun et al., 2014). Meta-analyses have revealed that individuals with dyslexia show atypical patterns of gray matter volume, cortical thickness, and white matter connectivity in brain regions implicated in language (Eckert et al., 2016; Mascheretti et al., 2017). Furthermore, KIAA0319 single nucleotide variants (SNVs) are associated with the prevalence of dyslexia and linked to differences in gray and white matter cortical organization, particularly within the posterior superior temporal lobe and parietal junction within the left hemisphere

\footnotetext{
Received April 17, 2021; accepted October 26, 2021; First published November 23, 2021.

The authors declare no competing financial interests.

Author contributions: W.D.H., B.J.B., and C.C.S. designed research; W.D.H., N.S., S.J.S., M.R., C.S., B.J.B., and C.S. performed research; W.D.H., N.S., M.M.M., and C.S. analyzed data; W.D.H., N.S., B.J.B., and C.C.S. wrote the paper.

This research was supported in part by National Institutes of Health Grants NS-092988, NS-73134, and HD-60563 and National Science Foundation INSPIRE Grant 1542848. The National Center for Chimpanzee Care (NCCC) chimpanzees are supported by the NIH NCRR Cooperative Agreement U42OD011197. The NCCC is fully accredited by AAALAC International.

Correspondence should be addressed to William D. Hopkins at wdhopkins@ mdanderson.org.

https://doi.org/10.1523/ENEURO.0169-21.2021

Copyright (C) 2021 Hopkins et al.
}

This is an open-access article distributed under the terms of the Creative Commons Attribution 4.0 International license, which permits unrestricted use, distribution and reproduction in any medium provided that the original work is properly attributed.
(Darki et al., 2012; Pinel et al., 2012; Jamadar et al., 2013; Eicher et al., 2016; Ozernov-Palchik and Gaab, 2016).

Studying the impact of variation in genes like KIAA0319 on primate brain morphology can provide insight into the evolution of human cognition and language systems by clarifying the functional role of genetic variation in shaping neural circuits. To this end, we tested whether chimpanzee KIAA0319 polymorphisms account for gray matter variation in brain regions implicated in language, particularly the posterior superior temporal and inferior frontal gyri. Comparative studies in nonhuman primates have shown that these regions play a role in the production and processing of species-specific communicative signals and vocalizations (Heffner and Heffner, 1984; Poremba et al., 2003, 2004; Gil-da-Costa et al., 2006; Petkov et al., 2008; Taglialatela et al., 2009). There is also evidence that chimpanzees, and to a lesser extent some monkeys, exhibit population-level morphologic and cellular asymmetries in the inferior frontal gyrus and superior temporal cortex, which overlap with Wernicke's area in the human brain (Hopkins and Nir, 2010; Spocter et al., 2010; Lyn et al., 2011; Marie et al., 2018), one of the main regions influenced by KIAA0319 polymorphisms. What remains unknown is whether individual variation in morphology or function in these brain regions is associated with KIAA0319 polymorphisms in chimpanzees.

To test this hypothesis, we initially identified the frequencies of KIAA0319 SNVs in a sample of unrelated chimpanzees. Next, we selected SNVs of interest for further genotype-phenotype study. We focused on SNVs present in the $5^{\prime}$ upstream region of the gene (likely important for gene regulation as they are in or near the promoter region and transcription start site of the gene), as well as nonsynonymous SNVs in the coding region of the gene (these cause amino acid changes and therefore are more likely to alter structure and/or function of the protein). Next, we genotyped these SNVs in a sample of chimpanzees for which in vivo T1-structural magnetic resonance imaging scans had previously been obtained. We used source-based morphometry (SBM) to examine whole-brain gray matter covariation differences across KIAA0319 SNVs. Additionally, we analyzed microstructural variation related to KIAA0319 polymorphisms in the posterior superior temporal cortex from histologic sections of postmortem chimpanzee brains.

\section{Materials and Methods}

\section{Subjects}

In the current study, we used archival structural T1weighted magnetic resonance imaging scans obtained from 136 adult captive chimpanzees ( 79 females and 57 males). Captive chimpanzees in the United States descended from a founder population that was $>95 \%$ from the western subspecies (Pan troglodytes verus; Ely et al., 
2005). The subjects were socially housed at the National Center for Chimpanzee Care (NCCC), which is part of The University of Texas MD Anderson Cancer Center. The NCCC facilities have indoor/outdoor enclosures with climbing structures, bedding, and daily environmental enrichment (Neal Webb et al., 2018). All chimpanzees had 24-h access to both indoor and outdoor areas except during cleaning. Care staff fed the chimpanzees a diet of commercially available primate chow and fresh produce multiple times per day, as well as several daily foraging opportunities and ad libitum access to water. All work was approved by the Institutional Animal Care and Use Committee at NCCC, followed the guidelines of the Institute of Medicine on the use of chimpanzees in research, and complied with the Society for Neuroscience Policy on Ethics.

\section{Identifying SNVs}

Initial quantification of genetic variation in chimpanzees was done using publicly available whole genome data [P. troglodytes troglodytes $N=18, P$. troglodytes verus $N=12, P$. troglodytes schweinfurthii $N=16, P$. troglodytes ellioti $N=10, P$. troglodytes (unknown subspecies) $N=3$; Prado-Martinez et al., 2013; De Manuel et al., 2016]. The genomes were mapped to the human genome (version hg19) using BWA-MEM v0.7.5a-r405 (http://bio-bwa. sourceforge.net/bwa.shtml) with default parameters. After removing duplicates using PICARD v1.91 (https:// sourceforge.net/projects/picard/files/picard-tools/1.91/), SNVs were called using GATK UnifiedGenotyper (https:// software.broadinstitute.org/gatk/documentation/tooldocs/ current/org_broadinstitute_gatk_tools_walkers_genotyper_ UnifiedGenotyper.php). Gene consensus sequences were built for each individual using vcfconsensus (http://vcftools. sourceforge.net/man_latest.html). Intronic regions were removed from the sequences and remaining promoter region and exons were aligned to the human KIAA0319 reference sequence (Ensembl: ENSG00000137261) using Geneious (version 6.0.6).

Next, to confirm the presence and allele frequencies of the genetic variants in the population of chimpanzees for which we have matching neuroanatomical data, we genotyped the SNVs that showed variation in genome data for $P$. troglodytes verus in an additional set of 25 presumed unrelated chimpanzees. Only variants that showed minor allele frequencies $>5 \%$ were then genotyped for the complete population. To infer the putative functional consequences of the resulting coding variants, we used SNAP2 (Hecht et al., 2015), a trained classifier based on a machine-learning device called a "neural network." It distinguishes between effect and neutral variants/nonsynonymous SNVs by taking a variety of sequence and variant features into account. The effect of a variant is believed to be of importance to the native protein function if the SNAP2 score exceeds 50 , is neutral if the score is below -50 and is unreliable when between 50 and -50 .

\section{DNA extraction and genotyping}

For the chimpanzees with matching neuroanatomical data, genomic DNA was extracted from 200- $\mu$ l blood samples using the QIAampDNA Mini kit automated on a QiaCube (QIAGEN), following the manufacturer's instructions. The DNA extract was recovered in $200 \mu \mathrm{l}$ of elution buffer and kept frozen at $-20^{\circ} \mathrm{C}$. Negative extraction controls showed no evidence of DNA contamination. DNA concentrations were quantified using a Nanodrop 2000 (Thermo-Fisher Scientific) spectrophotometer. Subsequent genotyping of the SNVs of interest was done using either Sanger sequencing or High-Resolution Melt Analysis (Smith et al., 2010). For exons where multiple SNVs were present in relatively short fragments $(<500 \mathrm{bp})$, exons were amplified using PCR. Primers were developed flanking the region with the SNVs (exon 4, forward CTT GGA AAC CAC CAG AAT CAT GCG G and reverse CTT TCT CCA ACA CCT CTC CTG AAG; exon 18, forward CTC AGC CCT ACA CAC CTC TTT $G$ and reverse AAG CCT CCT CTA TAC CAG AAC TC). The 25- $\mu$ I PCR mix contained $10.5 \mu \mathrm{l}$ MasterMix (QIAGEN; HotStarTaq plus DNA polymerase, deoxynucleotides, and $\mathrm{MgCl}_{2}$ ), $2.0 \mu \mathrm{l}$ primer mix (10 $\mathrm{mm}$ concentration of combined forward and reverse primers) and $\sim 5-20 \mathrm{ng}$ of genomic DNA (quantified via a NanoDrop microvolume spectrophotometer). PCR started with an initial activation at $95^{\circ} \mathrm{C}(5 \mathrm{~min})$, followed by 35 cycles of denaturing at $95^{\circ} \mathrm{C}(10 \mathrm{~s})$, annealing at $54^{\circ} \mathrm{C}(30 \mathrm{~s})$ and extension at $72^{\circ} \mathrm{C}(1 \mathrm{~min})$, with a final extension period of $10 \mathrm{~min}$ at $72^{\circ} \mathrm{C}$. All reactions included positive and no-template controls. The amplicons were then Sanger sequenced on an Applied Biosystems platform following manufacturer's specifications at the Yale DNA Analysis Facility. For $<100-b p$ exon sequences where only one SNV site was present, High-Resolution Melt Analysis (as in Jacobs et al., 2016) was used to assess genotypes. Primer pairs flanking the SNVs were designed to target a short segment ( $\sim 100 \mathrm{bp})$ containing the single polymorphic site (chr6:24899739, forward CTG ACT GAG ACT GGG AAC and reverse CTG TGG TIT AGA TTA TCA CG; chr6:24900865, forward GCA ATT CGT GAG GTT GGG and reverse GCC CTG AGT TCA GAA GG; chr6:24828663, forward GAA TGG AAA CCA GAG CAG TG and reverse ATG GAG AAC TTG CCT GCA TGA). All qPCRs and melting curves were generated on a Rotor-Gene Q (QIAGEN) platform. The $25 \mu \mathrm{L}$ qPCR mix contained $12.5 \mu \mathrm{L}$ HRM MasterMix (QIAGEN; HotStarTaq plus DNA polymerase, EvaGreen dye, Q-Solution, deoxynucleotides, and $\mathrm{MgCl}_{2}$ ), $1.75 \mu \mathrm{l}$ primer mix (10 mM concentration forward and reverse primers) and $\sim 20 \mathrm{ng}$ of genomic DNA (quantified via a NanoDrop microvolume spectrophotometer). qPCR started with an initial activation at $95^{\circ} \mathrm{C}(5 \mathrm{~min})$, followed by 40 cycles of denaturing at $95^{\circ}$ $\mathrm{C}(10 \mathrm{~s})$, annealing at $54^{\circ} \mathrm{C}(30 \mathrm{~s}) 72^{\circ} \mathrm{C}(40 \mathrm{~s})$ and a final extension period of $10 \mathrm{~min}$ at $72^{\circ} \mathrm{C}$. In each reaction, no-template controls were included. Data on melting curves and temperature of qPCR products were generated immediately following amplification by increasing temperatures from $65^{\circ} \mathrm{C}$ to $95^{\circ} \mathrm{C}$, rising by $0.1^{\circ} \mathrm{C} / 2 \mathrm{~s}$. Fluorescence data were plotted as a function of temperature during DNA denaturation (melting) and visualized and compared using the Rotor-Gene Q HRM software package (QIAGEN). The resulting melting temperatures and curve shapes were 
assigned to different genotypes based on previously established reference curves (as in Jacobs et al., 2016) of known variants. Genotypes were confirmed using multiple independent qPCR and high-resolution melt analyses (mean $=2.76$ replicates per sample). Additionally, for each melting curve-based genotype, we Sanger sequenced $10 \%$ of the samples to validate and confirm the genotype (Applied Biosystems Genetic Analyzer, DNA Analysis Facility at Yale University). Multiple alignments of the resulting DNA sequences were performed using Geneious (version 6.0.6).

\section{Magnetic resonance image acquisition}

We previously obtained magnetic resonance images using methods described elsewhere (Bogart et al., 2014; Bard and Hopkins, 2018; Mulholland et al., 2020). No new magnetic resonance images were collected for this study. Briefly, subjects were initially immobilized by injection of ketamine $(10 \mathrm{mg} / \mathrm{kg})$ or telazol $(2-6 \mathrm{mg} / \mathrm{kg})$ and subsequently anesthetized with propofol $(40-60 \mathrm{mg} / \mathrm{kg}$ ) per standard institutional guidelines. The subjects were then transported to the imaging facility and remained anesthetized for the duration of the scan (40-60 min depending on brain size), as well as during transportation back to a recovery cage ( $\sim 75-120 \mathrm{~min}$ in total). The subjects were placed in the scanner chamber in a supine position with their head inside the human-head coil. The chimpanzees were scanned using a 1.5-Tesla scanner and T1weighted images were collected in the transverse plane using a gradient echo protocol (pulse repetition $=19 \mathrm{~ms}$, echo time $=8.5 \mathrm{~ms}$, number of signals averaged $=8$, and a $256 \times 256$ matrix). After completing the magnetic resonance imaging scan collection, the subjects were temporarily housed in a single cage for several hours to allow them to recover from the anesthesia, after which they were returned to their social group.

\section{Postimage processing and analyses}

The archival magnetic resonance imaging data were processed on a Macintosh computer using previously described methods (Mulholland et al., 2020). First, we imported the raw DICOM files into 3D Slicer 4 (www. slicer.org) and converted each into NIfTI format (Fedorov et al., 2012; Kikinis et al., 2014). Next, we used the BET function in FSL for skull-stripping, using fractional intensity thresholds ranging between 0.35 and 0.80 (Smith, 2002; Jenkinson et al., 2005). The skull-stripped brains were then imported into 3D Slicer for N4ITK bias correction (spline distance $=50$, bias field $=0.15$, convergent threshold $=0.001$; Boyes et al., 2008; Tustison and Gee, 2009; Tustison et al., 2010, 2014). Using the MRI Denoising Package for MATLAB (R2015b; MathWorks), the scans were then denoised using an optimized nonlocal means denoising filter (Coupe et al., 2008). The scans were then resampled at 0.625 isotropic voxels and aligned in radiologic space in Analyze 11.0 (AnalyzeDirect), using a capsule placed during the imaging process as a left-right orientation marker. Finally, we used the FLIRT function in FSL to perform a 12-parameter affine linear registration (Jenkinson and Smith, 2001; Jenkinson et al., 2002) of the processed scan to a chimpanzee template brain (Hopkins and Avants, 2013).

We used the FSL-VBM pipeline (http://fsl.fmrib.ox.ac. $\mathrm{uk} / \mathrm{fs} /$ /fslwiki/FSLVBM) to process each preprocessed scan. The FSL-VBM pipeline included (1) segmentation of each scan into gray and white matter, (2) linear registration of each scan to a standard chimpanzee template (Hopkins and Avants, 2013), (3) creation of a study-specific gray matter template (Smith et al., 2004; Andersson et al., 2007; Douaud et al., 2007), (4) nonlinear registration of each subject's gray matter images to the study-specific template, (5) modulation of the gray matter volume by use of a Jacobian warp to correct for local expansion or contraction of gray matter within each voxel, and (6) smoothing with an isotropic Gaussian kernel with a $\sigma$ of $2 \mathrm{~mm}$.

\section{SBM and statistical analyses}

Following previously described methods (Hopkins et al., 2019; Mulholland et al., 2020), we subjected the individual modulated gray matter volume produced in the FSL-VBM pipeline to perform SBM using the software program GIFT (https://trendscenter.org/software/gift/ ). SBM is a relatively new method used to characterize gray and white matter structural covariation in samples of magnetic resonance imaging scans (AlexanderBloch et al., 2013; Bard and Hopkins, 2018). Unlike univariate analytic methods, such as voxel-based morphometry, SBM is a multivariate, data-driven analytic approach that utilizes information about relationships among voxels to group voxels carrying similar information across the brain. Without requiring prior determination of regions of interest, the resulting components or sources are identified based on the spatial information between voxels grouped in a natural manner and represent similar covariation networks between subjects; thus, this approach has been described as a multivariate version of voxel-based morphometry (Xu et al., 2009). At the individual level, GIFT outputs weighted scores for each component that reflect the relative contribution of each subject's gray matter volume to their generation. For this sample of chimpanzees, the SBM analysis yielded eight components. The weighted scores for each of the eight components were the dependent measures in the subsequent multivariate analysis of covariance (MANCOVA). Sex and KIAA0319 SNV alleles were the independent variables, while genetic relatedness was the covariate.

\section{Microstructural analyses of the posterior superior temporal cortex (area Tpt)}

In addition to the in vivo SBM analysis, we selected area Tpt for histologic analysis because it overlaps with one of the larger gray matter regions that differed between chimpanzees in the SBM analysis. For histologic sectioning, tissue blocks were frozen on dry ice, and cut at $40 \mu \mathrm{m}$ on a freezing sliding microtome. Every 10th section (spaced $400 \mu \mathrm{m}$ apart) from each block 
was mounted on chromalum-subbed slides, stained using $0.5 \%$ cresyl violet to visualize cytoarchitecture, and coverslipped with DPX.

We quantified the neuropil fraction from the posterior superior temporal cortex (area Tpt) in the postmortem brains of 15 chimpanzees (ages 12-48 years, mean 32.6 years; seven female, eight male). The neuropil is defined as the space between neuronal and glial cell bodies, which is comprised of dendrites, axons, synapses, and microvasculature (Spocter et al., 2012). The neuropil fraction data came from previously published data in 12 individuals from Spocter et al. (2012), supplemented by an additional three brains measured for the current study, which had been processed more recently for histology. Neuropil fraction was measured using high-resolution images of these Nissl-stained sections. Imaging of the regions of interest was performed with a Zeiss Axioplan 2 photomicroscope (Zeiss) equipped with a Ludi XY motorized stage (Ludl Electronics), Heidenhain $z$-axis encoder, and an Optronics MicroFire color video camera coupled to a Dell PC using Stereoinvestigator software (MBF Bioscience). For each chimpanzee brain, three evenly spaced coronal sections were sampled throughout the region of interest. For each section, regions of interest were contoured under low magnification (2.5× objective lens) underneath a representative portion of the area (for cortical regions this was $\sim 3 \mathrm{~mm}$ in length along the cortical surface). At least 30 systematically random sampled images were taken within the contours of each section using a $20 \times$ objective lens, resulting in images at 0.53 pixels $/ \mu \mathrm{m}$ resolution. Each image was imported into ImageJ (v.1.32j) and subjected to background subtraction with a rolling ball radius of 50 pixels and then converted to binary by an automated threshold routine (Spocter et al., 2012). Before calculation of the neuropil fraction, images that contained artifacts were removed from the batch and the remaining images were blind-coded to avoid observer bias.

We also analyzed previously published data on regional volume, total neuron number, and neuron density from area Tpt in 12 chimpanzees (Spocter et al., 2010). In brief, stereologic methods were used, including Cavalieri point counting to calculate the regional volumes of area Tpt, and optical fractionator sampling to estimate neuron numbers. In the cresyl violet Nissl-stained sections, neurons were distinguished from glial cells by the presence of a large, lightly stained nucleus and a distinct nucleolus, accompanied by lightly stained dendritic processes. Stereologic counts of neuron numbers based on cresyl violet Nissl-staining have been shown to produce results that are highly correlated with other methods of labeling neurons, such as NeuN immunostaining (Zhu et al., 2015).

We ran a series of multivariate general linear models using Type III sum of squares with each dependent measure of cortical area Tpt neuropil fraction, regional volume, neuron number, and neuron density in the left and right hemisphere, with sex, age, and brain mass as covariates, and all three KIAA0319 genotypes as fixed factors. We also analyzed the asymmetry quotient (AQ; right - left/average of hemispheres) of neuropil fraction using general linear models with sex, age, and brain mass as covariates.

\section{Results}

\section{KIAA0319 genetic variation}

We identified 33 SNVs in chimpanzee KIAA0319 5' flanking region and coding regions combined (see Table 1). Out of 33 SNVs, four were in the upstream flanking region and 29 were in the amino acid coding region. A total of 18 out of 29 SNVs were nonsynonymous variants, and only two of these were present in $P$. troglodytes verus: chr6:24841281: C/A in exon 4 resulting in a Val295Phe substitution, and chr6:24828663: $A / C$ in exon 10 resulting in a Glu563Asp substitution. Next, we genotyped these two SNVs in our population of chimpanzees with matching neuroanatomical data and found that only chr6:24828663: A/C showed minor allele frequencies $>5 \%$ and was thus suitable for further genotype-phenotype analysis. Genotype frequencies for this SNV (hereafter referred to as KIAA0391 Glu563Asp) were in Hardy Weinberg equilibrium $\left(\chi^{2}=0.52, \mathrm{df}=1\right.$, $p=0.47$ ) and SNAP2 prediction of its functional consequences resulted in a modest SNAP2 effect score of 35 (expected accuracy $66 \%$ ).

Out of the four SNVs present in the promoter flanking region, two were in complete linkage disequilibrium in our chimpanzee population (chr6:24899739:T/A and chr6:24899753:C/A), therefore only one was included in further genotype-phenotype analysis. A third SNV (chr6:24899741:C/G) did not exceed the $>5 \%$ minor allele threshold and was also excluded from further analysis. For the two promoter region flanking region SNVs that were included in the analysis, genotype frequencies were in Hardy Weinberg equilibrium (chr6:24899739:T/ A $\chi^{2}=3.79$, df $=1, p=0.05$; chr6:24900865:A/G $\chi^{2}=2.84$, $\mathrm{df}=1, p=0.09$; hereafter referred to as KIAA0391rsP1 and KIAA0391rsP4, respectively). The specific SNVs used in the subsequent analyses are highlighted in Table 1.

\section{KIAA0319 and gray matter covariation}

For KIAA0319rsP1, the MANCOVA revealed a significant main effect for genotype $F_{(16,246)}=2.086, p=0.01$. Subsequent univariate $F$-tests revealed significant differences between the alleles for SBM component 1 (C1) $F_{(2,129)}=4.186, p=0.017$ and component 2 (C2) $F_{(2,129)}=$ 6.394, $p=0.002$ (Fig. 1A). Post hoc analysis for $\mathrm{C} 1$ indicated that chimpanzees with the AA allele had higher weighted scores compared with AT and $T T$ individuals, but the AT and TT means did not differ significantly from each other. For $\mathrm{C} 2$, post hoc analysis indicated that the means for the AA, AT, and TT groups all differed significantly from each other (Fig. 1B). No other SBM components differed across the KIAA0319rsP1 alleles. For KIAA0319rsP4, the results were very similar. The MANCOVA revealed a significant main effect for KIAA0319 genotype $F_{(16,244)}=2.790, p=0.007$. Univariate $F$-tests revealed significant main effects of the SNV genotype on $C 1 F_{(2,128)}=3.810$, $p=0.025$ and $\mathrm{C} 2 F_{(2,128)}=5.884, p=0.004$ (Fig. $1 A$ ). Post hoc analysis for component $\mathrm{C} 1$ indicated that chimpanzees with 
Table 1: Chimpanzee KIAA0319 genetic variation

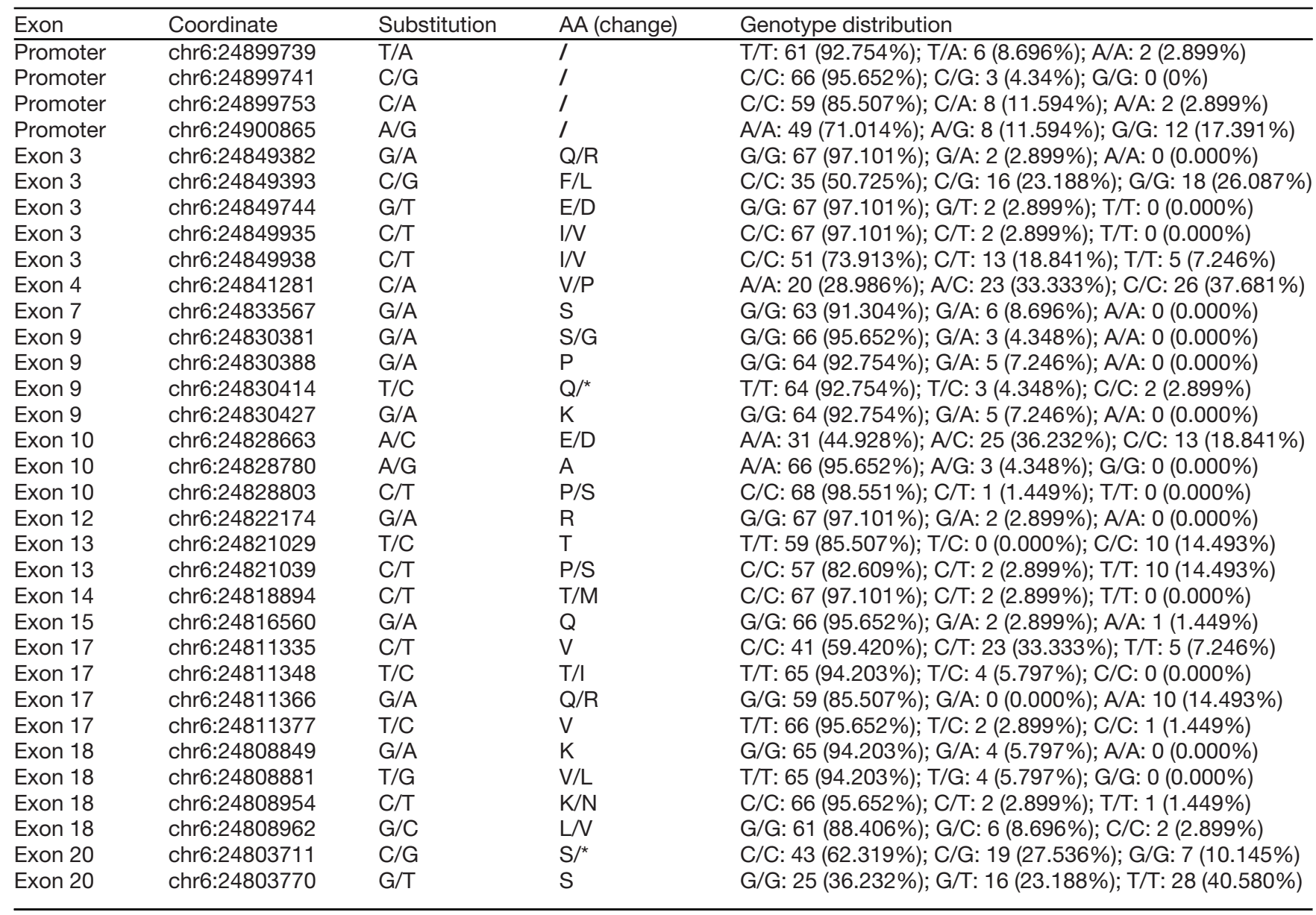

Coordinates are shown in reference to Pantro 4 (UCSC genome browser).

Table 2: Mean and AQ TpT total neuron, neuron density, volume, and neuropil space values (SE) for chimpanzees with different KIAA0319rsP1 alleles

\begin{tabular}{llll}
\hline KIAA0319_rsP1 & AA & Genotype & \\
\hline Average & & AT & TT \\
Total neuron & 10118976 & 10931286 & 14375043 \\
& $(3616305)$ & $(3275682)$ & $(3531981)$ \\
Neuron density & 29311 & 29926 & 37084 \\
& $(4392)$ & $(3684)$ & $(4852)$ \\
Volume & 355 & 368 & 390 \\
Neuropil space & $(80)$ & $(73)$ & $(79)$ \\
& 0.696 & 0.680 & 0.652 \\
Asymmetry (AQ) & $(0.04)$ & $(0.03)$ & $(0.04)$ \\
Total neuron & & & \\
& -0.409 & -0.517 & -0.263 \\
Neuron density & $(0.31)$ & $(0.28)$ & $(0.31)$ \\
& -0.225 & -0.314 & +0.132 \\
Volume & $(0.19)$ & $(0.16)$ & $(0.21)$ \\
& -0.16 & -0.253 & -0.352 \\
Neuropil space & $(0.31)$ & $(0.28)$ & $(0.30)$ \\
& 0.066 & 0.010 & -0.069 \\
& $(0.03)$ & $(0.03)$ & $(0.03)$ \\
\hline
\end{tabular}

the minor AA allele had lower weighted scores compared with GG, but not GT individuals. By contrast, for C2, post hoc analysis indicated that $A A$ had significantly higher values than GG but did not differ from AG apes (Fig. 1C). No other SBM components differed across the KIAA0319rsP4 alleles. For the KIAA0319 Glu563Asp variant, the MANCOVA revealed no significant main effects or interactions. Notwithstanding, the univariate $F$-tests revealed a significant main effect for the genotype on C7 $F_{(2,1298)}=3.980, p=0.046$ (Fig. 2A). Chimpanzees with the major $A C$ allele had lower weighted scores than individuals with the CC but not AA allele (Fig. 2B). No other SBM components differed across the KIAA0319 Glu563Asp alleles. (Note, see Fig. 3 for a chimpanzee atlas map; Vickery et al., 2020.)

\section{KIAA0319 and microstructure in posterior superior temporal cortex (area Tpt)}

We ran a series of multivariate general linear models with each dependent measure of cortical area Tpt microstructure in the left and right hemisphere with sex, age, and brain mass as covariates, and all three KIAA0319 genotypes as fixed factors. Of these models, the only effect that approached a conventional statistical threshold was the KIAA0319rsP1 genotype on right hemisphere neuropil 


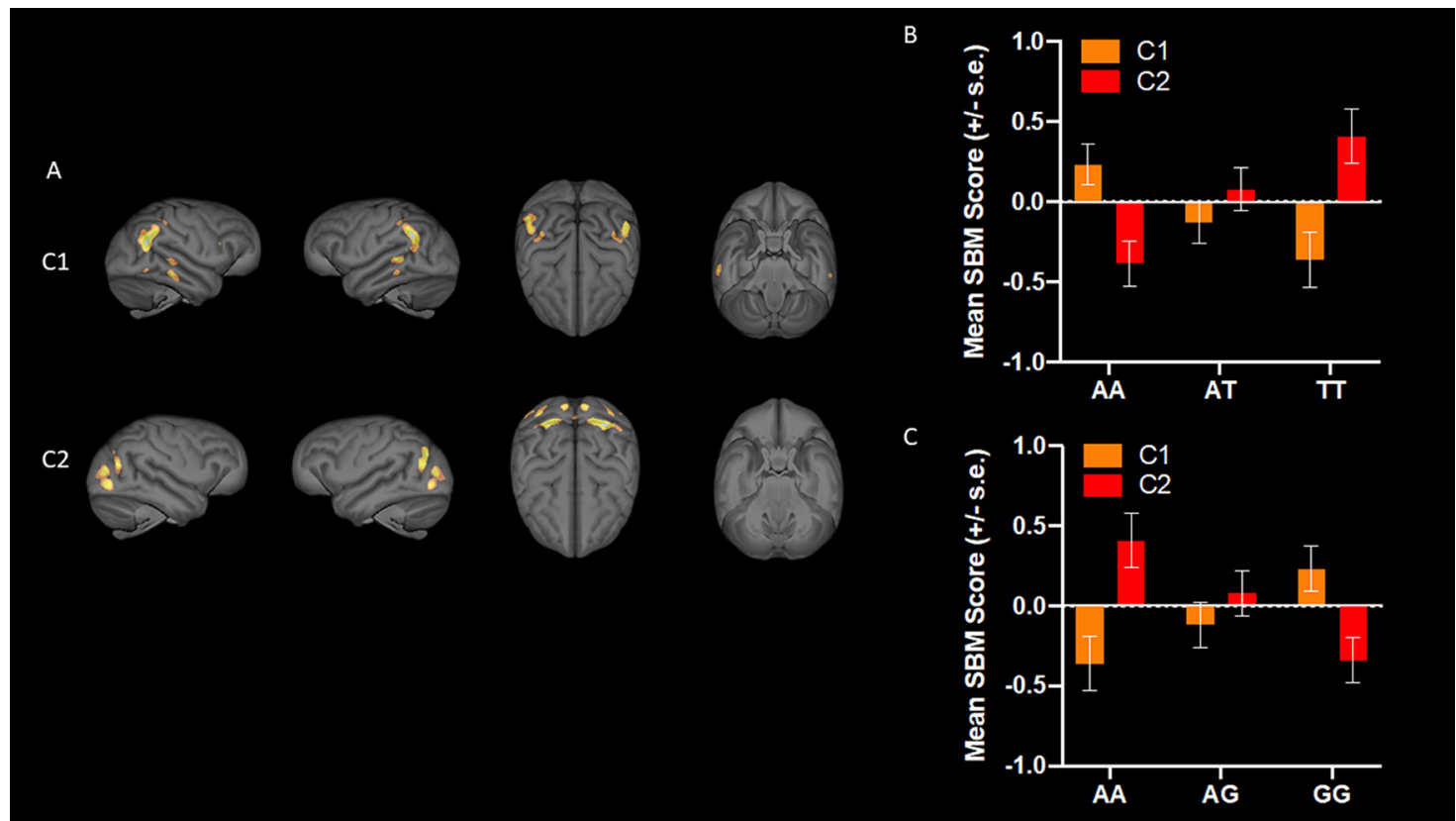

Figure 1. $\boldsymbol{A}, 3 \mathrm{D}$ renderings of SBM C1 and $\mathrm{C} 2$ (from left to right: right, left, top, and bottom views), and mean ( \pm SE) SBM weighted scores for each allele of $(\boldsymbol{B})$ KIAA0319rsP1 and (C) KIAA0319rsP4. B, Chimpanzees with the AA allele had higher weighted SBM scores on $\mathrm{C} 1$ compared with AT and TT individuals but the AT and TT means did not differ significantly from each other. For component two, AA, AT, and TT groups all differed significantly from each other. $C$, For KIAA0319rsP4, chimpanzees with the minor AA allele had lower weighted SBM scores for C1 compared with GG but not GT individuals. By contrast, for C2, chimpanzees with AA had significantly higher values than GG but did not differ from AG chimpanzees.

fraction; $F_{(2,10)}=10.266, p=0.089$ ) (see Table 2). The covariates sex, age, and brain mass did not have significant between-subjects effects. To further examine lateralization in cortical area Tpt microstructure, we also analyzed the $A Q$ of each variable using general linear models with sex, age, and brain mass as covariates. Of these models, the only effect that approached a conventional statistical threshold was the KIAA0319rsP1 genotype on AQ of neuropil fraction; $F_{(2,10)}=14.553, p=0.064$. The covariates sex, age, and brain mass did not have significant between-subjects effects. Photomicrographs and results can be seen in Figure 4.

\section{Discussion}

Exploration of polymorphic variation in KIAA0319 revealed a relatively variable coding sequence in chimpanzees. Across all four sub-species included in this study, a total of 29 SNVs were found spread across 12 of the 20 exons of the gene. Of these, a majority were nonsynonymous, thus causing amino acid changes, indicating low structural stability of the receptor protein in chimpanzees. Only a small proportion of SNVs were present in the flanking region $(N=4)$. This is in line with previous research showing that the most pronounced conservation across species in KIAA0319 extends from the transcription start

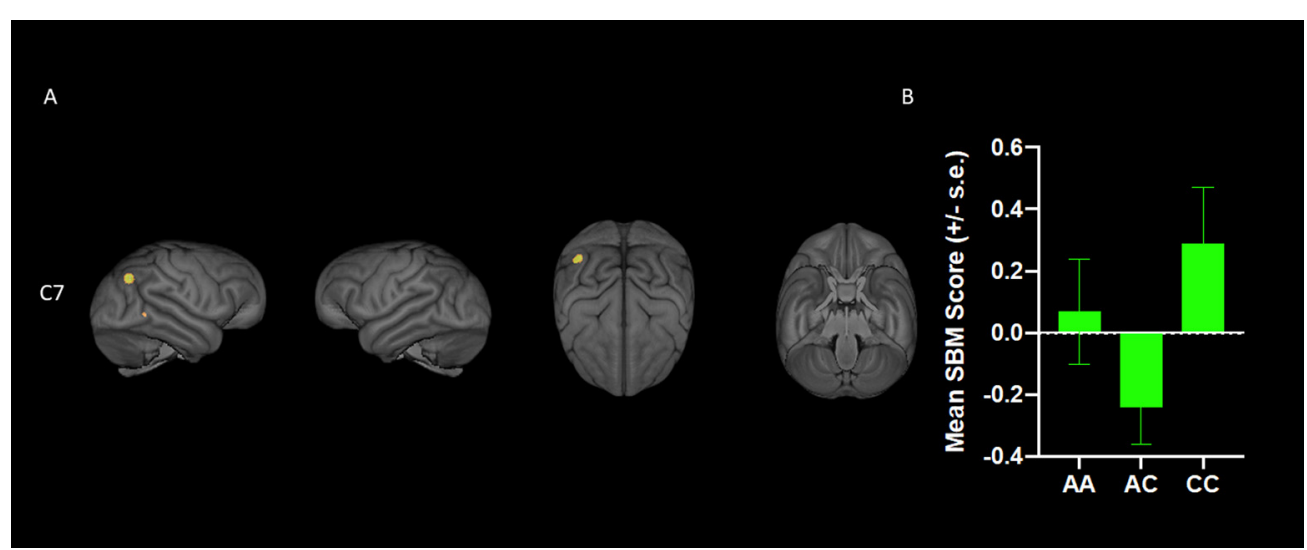

Figure 2. $\boldsymbol{A}, 3 \mathrm{D}$ renderings of SBM component 7 (from left to right: right, left, top, and bottom views). $\boldsymbol{B}$, Mean ( \pm SE) SBM weighted scores for each allele of the KIAA0319 Glu563Asp variant. Chimpanzees with the major AC allele had lower weighted SBM scores for component 7 than individuals with the CC but not AA allele. 


$\begin{array}{ll}2 & \text { Anterior Superior Frontal G. } \\ 4 & \text { Middle Superior Frontal G. } \\ 6 & \text { Posterior Superior Frontal G. } \\ 8 & \text { Anterior Middle Frontal G. } \\ 10 & \text { Posterior Middle Frontal G. } \\ 12 & \text { Anterior Inferior Frontal G. } \\ 14 & \text { Middle Inferior Frontal G. } \\ 16 & \text { Posterior Inferior Frontal G. } \\ 18 & \text { Medial Orbitofrontal Cortex } \\ 20 & \text { Lateral Orbitofrontal Cortex } \\ 22 & \text { Anterior Cingulate Cortex } \\ 24 & \text { Middle Cingulate Cortex } \\ 26 & \text { Posterior Cingulate Cortex } \\ 28 & \text { Superior Precentral G. } \\ 30 & \text { Middle Precentral G. } \\ 32 & \text { Inferior Precentral G. } \\ 34 & \text { Paracentral Lobule } \\ 36 & \text { Frontal Operculum } \\ 38 & \text { Parietal Operculum } \\ 40 & \text { Anterior Insula } \\ 42 & \text { Posterior Insula } \\ 44 & \text { Anterior Transverse Temporal G. } \\ 46 & \text { Posterior Transverse Temporal G. } \\ 48 & \text { Anterior Superior Temporal G. } \\ 50 & \text { Posterior Superior Temporal G. } \\ 52 & \text { Anterior Middle Temporal G. } \\ 54 & \text { Posterior Middle Temporal G. } \\ 56 & \text { Anterior Inferior Temporal G. } \\ 58 & \text { Posterior Inferior Temporal G. } \\ 60 & \text { Entorhinal Cortex } \\ 62 & \text { Anterior Fusiform G. } \\ 64 & \text { Posterior Fusiform G. } \\ & \end{array}$

\section{Davi130 Parcellation}

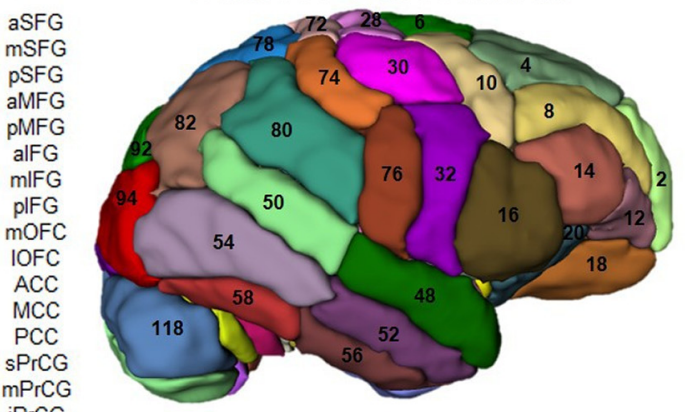

iPrCG

PCL

FOP
POP

alns

plns

aTTG

pITG

aSTG

PSTG

aMTG

pMTG

alTG

pITG

EnC

pFFG

\begin{tabular}{|c|c|c|}
\hline 68 & $\begin{array}{l}\text { Parahıppocampal G. } \\
\text { Amygdala }\end{array}$ & $\begin{array}{l}\text { PHC } \\
\text { Amy }\end{array}$ \\
\hline 70 & Hippocampus & $\mathrm{HC}$ \\
\hline 72 & Superior Postcentral G. & sPoCG \\
\hline 74 & Middle Postcentral G. & $\mathrm{mPoCG}$ \\
\hline 76 & Inferior Postcentral G. & iPoCG \\
\hline 78 & Superior Parietal Lobule & SPL \\
\hline 80 & Supramarginal G. & SMG \\
\hline 82 & Angular $\mathrm{G}$. & AnG \\
\hline 84 & Precuneus & PCun \\
\hline 86 & Cuneus & Cun \\
\hline 88 & Lingual G. & LG \\
\hline 90 & Calcarine Sulcus & Calc \\
\hline 92 & Superior Occipital G. & sOG \\
\hline 9 & Middle Occipital G. & $\mathrm{mOG}$ \\
\hline 96 & Inferior Occipital G. & iOG \\
\hline 98 & Caudate Nuclues & $\mathrm{CN}$ \\
\hline 100 & Nucleus Accumbens & NA \\
\hline & Basal Forebrain Nuclei & $\mathrm{BF}$ \\
\hline & Putamen & $\mathrm{Pu}$ \\
\hline 106 & Globus pallidus & GF \\
\hline 108 & Thalamus & Th \\
\hline 110 & Hypothalamus & HTh \\
\hline 112 & Cerebellum IX - Tonsil & CerlX \\
\hline 114 & Cerebellum VIIIAB & CerVIII \\
\hline 116 & Cerebellum VIIA - Crus I & Crusl \\
\hline 118 & Cerebellum VIIA - Crus II & Crusll \\
\hline 12 & Cerebellum VI & CerVI \\
\hline 122 & Cerebellum V-Lobe B & CerVB \\
\hline & Cerebellum V-Lobe A & CerVA \\
\hline & Cerebellum IV & CerlV \\
\hline & Cerebellum III & Cerlll \\
\hline & Cerebellum II & Cerll \\
\hline
\end{tabular}

Figure 3. Lateral and medial views of the right hemisphere Davi130 parcellation of the chimpanzee brain, region numbers correspond to names in the figure. Reprinted with permission (Vickery et al., 2020).

site to $\sim 1 \mathrm{~kb}$ upstream (Dennis et al., 2009). In humans, several SNVs in the upstream region of the gene have been shown to have strong associations with reading disorders and dyslexia (Cope et al., 2005; Harold et al., 2006; Dennis et al., 2009; Elbert et al., 2011) and similarly, in our study, the strongest association with brain phenotypic differences was found for variants KIAA0319rsP1 and KIAA0319rsP4, despite a lack of overlap between human and chimpanzee promoter region SNVs. It thus appears for our chimpanzee population, that differential gene expression patterns are a likely driving factor behind this phenotypic variation, but further studies using gene expression assays are required to confirm this conclusion.

Findings from the SBM analysis showed that two SNVs in the promotor region of the KIAA0319 gene (KIAA0319rsP1 and KIAA0319rsP4), were associated with differences in gray matter volume of several brain regions. As hypothesized, these polymorphisms were associated with differences in gray matter covariation of the posterior superior temporal gyrus (including area Tpt and Heschl's gyrus, HG). This brain region is associated with a variety of cognitive functions including language comprehension and production and previous studies have reported that atypical language processing is associated with SNVs in KIAA0319 in humans (Hirayasu et al., 2000; Goulven and Tzourio-mazoyer, 2004; Dorsaint-Pierre et al., 2006; Warrier et al., 2009; Darki et al., 2012; Pinel et al., 2012; Jamadar et al., 2013; Friederici, 2015; Cardin et al., 2016; Eicher et al., 2016; Ozernov-Palchik and Gaab, 2016; Skeide et al., 2016).

The evidence that SNVs in KIAA0319 are associated with variation in gray matter within the posterior superior temporal gyrus in chimpanzees is a somewhat paradoxical finding in light of the evidence of its role in language and reading, aptitudes that are arguably uniquely human. In fact, Dumas et al. (2021) recently reported that KIAA0319 is under strong positive selection in humans compared with primate ancestors, and that this gene (and other communication-related genes) only diverged within the hominin lineage. At the most parsimonious level, the findings reported here suggest that although SNVs in KIAA0319 are directly implicated in the morphology of the posterior superior temporal gyrus, their link to the mapping of linguistic functions onto these brain regions is less direct. In other words, SNVs in KIAA0319 may be associated with both Tpt and HG morphology and specific kinds of language impairments, but the association is not causal, but rather mediating of other factors related to experience. Alternatively, the results reported here may indicate that there are more common neuropsychological processes that underlie individual variation in auditory-visual learning in human and nonhuman primates that are similarly influenced by SNV variation in KIAA0319 in both taxa. For instance, studies on statistical learning have shown that human and nonhuman primates are sensitive to temporal regularities in auditory and visual signals, which some have argued is the foundation for speech as well as syntactical processing (Hauser et al., 2001; Newport et al., 2004; Petkov and Jarvis, 2012; Fagot, 2017; Heimbauer et al., 2018). In great apes, several studies have reported impressive human speech comprehension abilities, including the processing of sentences presented in English only (Savage-Rumbaugh et al., 1993; Sevcik and Savage-Rumbaugh, 1994; Brakke and Savage-Rumbaugh, 1995). Finally, neuroimaging and lesion studies have shown that area Tpt, HG, and a number of other regions are involved in processing of 

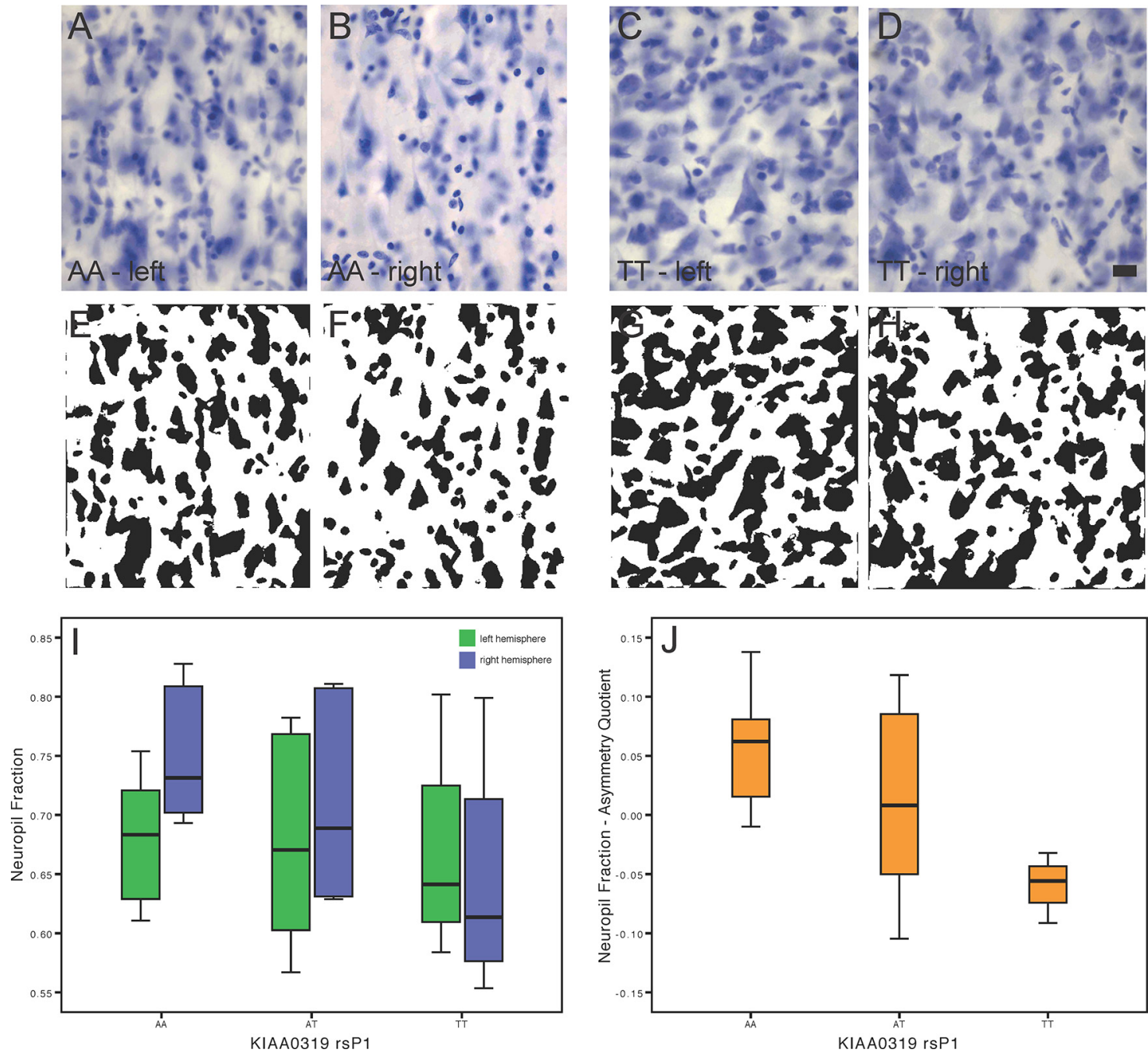

Figure 4. Hemispheric differences in neuropil fraction from posterior superior temporal cortex (area Tpt) of chimpanzees genotyped for KIAA0319rsP1. Photomicrographs of Nissl-stained sections from Layer III of area Tpt in an AA genotype chimpanzee's left $(\boldsymbol{A})$ and right $(\boldsymbol{B})$ hemisphere, and a TT genotype chimpanzee's left $(\boldsymbol{C})$ and right $(\boldsymbol{D})$ hemisphere. After background correction, the image frames are converted to binary for measurement of neuropil fraction $(\boldsymbol{E}-\boldsymbol{H})$ where the black pixels represent cell profiles and white pixels represent the neuropil space. Results from the sample are displayed as box and whisker plots of the distribution of neuropil fraction values between hemispheres $(\boldsymbol{I})$ and the AQ $(\boldsymbol{J})$ according to KIAA0319 rsP1 genotype.

species-specific vocalizations (Heffner and Heffner, 1984; Poremba et al., 2003, 2004; Gil-da-Costa et al., 2006; Petkov et al., 2008; Taglialatela et al., 2009). Based on these collective findings, it is intriguing to speculate that SNVs in KIAA0319 in nonhuman primates may underlie individual variation in auditory-visual learning and associated brain regions, although this hypothesis awaits additional research.

In addition, our microstructure analysis showed that KIAA0319rsP1 variants are associated with hemispheric differences in neuropil fraction of chimpanzees. This suggests that KIAA0319 expression may be involved in regulation of processes related to the formation and maintenance of synapses, dendrites, or axons in the microstructure of chimpanzee brains within a region directly involved in communication. Studies in rodents have shown that KIAA0319 plays a role in neural migration during formation of the cerebral neocortex, the development of dendritic arbors and spines, axon outgrowth, and has functional impacts on auditory processing (Peschansky et al., 2010; Platt et al., 2013; Centanni et al., 2014; Franquinho et al., 2017; Guidi et al., 2017). However, there are limited data examining KIAA0319 expression in the brains of humans or other primates (Kato et al., 2014; Muntané et al., 2017). It should be noted that our findings are based on a relatively small sample size, as is common for postmortem histologic studies. Nevertheless, in combination with the SBM results, these microstructure data further support the conclusion that KIAA0319 influences neuroanatomy in a manner that is likely to be functionally significant. Future studies are needed to address whether the hemispheric differences in neuropil fraction are because of variation in synapses, dendritic morphology, or axon distributions. 


\section{References}

Alexander-Bloch A, Giedd JN, Bullmore E (2013) Imaging structural co-variance between human brain regions. Nat Rev Neurosci 14:322-336.

Andersson JL, Jenkinson M, Smith S (2007) Non-linear registration aka spatial normalization. FMRIB technical report TR07JA2. Oxford: FMRIB Analysis Group of the University of Oxford.

Bard KA, Hopkins WD (2018) Early socioemotional intervention mediates long-term effects of atypical rearing on structural covariation in gray matter in adult chimpanzees. Psychol Sci 29:594-603.

Bogart SL, Bennett AJ, Schapiro SJ, Reamer LA, Hopkins WD (2014) Different early rearing experiences have long-term effects on cortical organization in captive chimpanzees (Pan troglodytes). Dev Sci 17:161-174.

Boyes RG, Gunter JL, Frost C, Janke AL, Yeatman T, Hill DLG, Bernstein MA, Thompson PM, Weiner MW, Schuff N, Alexander GE, Killiany RJ, DeCarli C, Jack CR, Fox NC (2008) Intensity nonuniformity correction using N3 on 3-T scanners with multichannel phased array coils. Neuroimage 39:1752-1762.

Brakke KE, Savage-Rumbaugh ES (1995) The development of language skills in bonobo and chimpanzee. I. Comprehension. Lang Commun 15:121-148.

Cardin V, Smittenaar RC, Orfanidou E, Rönnberg J, Capek CM, Rudner M, Woll B (2016) Differential activity in Heschl's gyrus between deaf and hearing individuals is due to auditory deprivation rather than language modality. Neuroimage 124:96-106.

Centanni TM, Booker AB, Sloan AM, Chen F, Maher BJ, Carraway RS, Khodaparast N, Rennaker R, LoTurco JJ, Kilgard MP (2014) Knockdown of the dyslexia-associated gene Kiaa0319 impairs temporal responses to speech stimuli in rat primary auditory cortex. Cereb Cortex 24:1753-1766.

Cope N, Harold D, Hill G, Moskvina V, Stevenson J, Holmans P, Owen MJ, O'Donovan MC, Williams J (2005) Strong evidence that KIAA0319 on chromosome $6 p$ Is a susceptibility gene for developmental dyslexia. Am J Hum Genet 76:581-591.

Coupe P, Yger P, Prima S, Hellier P, Kervrann C, Barillot C (2008) An optimized blockwise nonlocal means denoising filter for 3-D magnetic resonance images. IEEE Trans Med Imaging 27:425-441.

Darki F, Peyrard M, Matsson H, Kere J, Klingberg T (2012) Three dyslexia susceptibility genes, DYX1C1, DCDC2, and KIAA0319, affect temporo-parietal white matter structure. Biol Psychiatry 72:671676.

de Manuel M, Kuhlwilm M, Frandsen P, Sousa VC, Desai T, PradoMartinez J, Hernandez-Rodriguez J, Dupanloup I, Lao O, Hallast P, Schmidt JM, Heredia-Genestar JM, Benazzo A, Barbujani G, Peter BM, Kuderna LFK, Casals F, Angedakin S, Arandjelovic M, Boesch C, et al. (2016) Chimpanzee genomic diversity reveals ancient admixture with bonobos. Science 354:477-481.

Dennis MY, Paracchini S, Scerri TS, Prokunina-Olsson L, Knight JC, Wade-Martins R, Coggil P, Beck S, Green ED, Monaco AP (2009) A common variant associated with dyslexia reduces expression of the KIAA0319 gene. PLoS Genet 5:e1000436.

Dorsaint-Pierre R, Penhune VB, Watkins KE, Neelin P, Lerch JP, Bouffard M, Zatorre RJ (2006) Asymmetries of the planum temporale and Heschl's gyrus: relationship to language lateralization. Brain 129:1164-1176.

Douaud G, Smith S, Jenkinson M, Behrens T, Johansen-Berg $H$, Vickers J, James S, Voets N, Watkins K, Matthews PM, James A (2007) Anatomically related grey and white matter abnormalities in adolescent-onset schizophrenia. Brain 130:2375-2386.

Dumas G, Malesys S, Bourgeron T (2021) Systematic detection of brain protein-coding genes under positive selection during primate evolution and their roles in cognition. Genome Res 31:484-496.

Eckert MA, Berninger VW, Vaden KI Jr, Gebregziabher M, Tsu L (2016) Gray matter features of reading disability: a combined meta-analytic and direct analysis approach $(1,2,3,4)$. eNeuro 3 : ENEURO.0103-15.2015.

Eicher JD, Montgomery AM, Akshoomoff N, Amaral DG, Bloss CS, Libiger O, Schork NJ, Darst BF, Casey BJ, Chang L, Ernst T,
Frazier J, Kaufmann WE, Keating B, Kenet T, Kennedy D, Mostofsky S, Murray SS, Sowell ER, Bartsch H, et al. (2016) Dyslexia and language impairment associated genetic markers influence cortical thickness and white matter in typically developing children. Brain Imaging Behav 10:272-282.

Elbert A, Lovett MW, Cate-Carter T, Pitch A, Kerr EN, Barr CL (2011) Genetic variation in the KIAA0319 5' region as a possible contributor to dyslexia. Behav Genet 41:77-89.

Ely JJ, Dye B, Frels WI, Fritz J, Gagneux P, Khun HH, Switzer WM, Lee DR (2005) Subspecies composition and founder contribution of the captive US chimpanzee (Pan troglodytes) population. Am J Primatol 67:223-241.

Enard W, Przeworski M, Fisher SE, Lai CSL, Wiebe V, Kitano T, Monaco AP, Pääbo S (2002) Molecular evolution of FOXP2, a gene involved in speech and language. Nature 418:869-872.

Fagot $J$ (2017) Orthographic processing in animals: implications for comparative psychologists. Learn Behav 45:209-210.

Fedorov A, Beichel R, Kalpathy-Cramer J, Finet J, Fillion-Robin JC, Pujol S, Bauer C, Jennings D, Fennessy F, Sonka M, Buatti J, Aylward S, Miller JV, Pieper S, Kikinis R (2012) 3D slicer as an image computing platform for the quantitative imaging network. Magn Reson Imaging 30:1323-1341.

Fisher SE, Marcus GF (2006) The eloquent ape: genes, brains and the evolution of language. Nat Rev Genet 7:9-20.

Fisher SE, Scharff C (2009) FOXP2 as a molecular window into speech and language. Trends Genet 25:166-177.

Franquinho F, Nogueira-Rodrigues J, Duarte JM, Esteves SS, Carter-Su C, Monaco AP, Molnár Z, Velayos-Baeza A, Brites P, Sousa MM (2017) The dyslexia-susceptibility protein KIAA0319 inhibits axon growth through Smad2 signaling. Cereb Cortex 27:1732-1747.

Friederici AD (2015) White-matter pathways for speech and language processing. Handb Clin Neurol 129:177-186.

Gil-da-Costa R, Martin A, Lopes MA, Muñoz M, Fritz JB, Braun AR (2006) Species-specific calls activate homologs of Broca's and Wernicke's areas in the macaque. Nat Neurosci 9:1064-1070.

Goulven J, Tzourio-mazoyer N (2004) Hemispheric specialization for language. Brain Res Brain Res Rev 44:1-12.

Guidi LG, Mattley J, Martinez-Garay I, Monaco AP, Linden JF, Velayos-Baeza A, Molnár Z (2017) Knockout mice for dyslexia susceptibility gene homologs KIAA0319 and KIAA0319L have unaffected neuronal migration but display abnormal auditory processing. Cereb Cortex 27:5831-5845.

Harold D, Paracchini S, Scerri T, Dennis M, Cope N, Hill G, Moskvina V, Walter J, Richardson AJ, Owen MJ, Stein JF, Green ED, O'Donovan MC, Williams J, Monaco AP (2006) Further evidence that the KIAA0319 gene confers susceptibility to developmental dyslexia. Mol Psychiatry 11:1085-1091.

Hauser MD, Newport EL, Aslin RN (2001) Segmentation of the speech stream in a non-human primate: statistical learning in cotton-top tamarins. Cognition 78:B53-B64.

Hecht M, Bromberg Y, Rost B (2015) Better prediction of functional effects for sequence variants. BMC genomics 16:S1.

Heffner HE, Heffner RS (1984) Temporal lobe lesions and perception of species-specific vocalizations by macaques. Science 226:7576.

Heimbauer LA, Conway CM, Christiansen MH, Beran MJ, Owren MJ (2018) Visual artificial grammar learning by rhesus macaques (Macaca mulatta): exploring the role of grammar complexity and sequence length. Anim Cogn 21:267-284.

Hirayasu Y, McCarley RW, Salisbury DF, Tanaka S, Kwon JS, Frumin M, Snyderman D, Yurgelun-Todd DA, Kikinis R, Jolesz FA, Shenton ME (2000) Planum temporale and Heschl gyrus volume reduction in schizophrenia. Arch Gen Psychiatry 57:692-699.

Hopkins WD, Nir T (2010) Planum temporale surface area and grey matter asymmetries in chimpanzees (Pan troglodytes): the effect of handedness and comparison with findings in humans. Behav Brain Res 208:436-443. 
Hopkins WD, Avants BB (2013) Regional and hemispheric variation in cortical thickness in chimpanzees (Pan troglodytes). J Neurosci 33:5241-5248

Hopkins WD, Latzman RD, Mareno MC, Schapiro SJ, Gómez-Robles A, Sherwood CC (2019) Heritability of gray matter structural covariation and tool use skills in chimpanzees (Pan troglodytes): a source-based morphometry and quantitative genetic analysis. Cereb Cortex 29:3702-3711.

Jacobs RL, Baden AL, Spriggs A, MacFie TS, Irwin MT, Wright PC, Louis EE Jr, Lawler RR, Mundy NI, Bradley BJ (2016) Primate genotyping via high resolution melt analysis: rapid and reliable identification of color vision status in wild lemurs. Primates 57:541-547.

Jamadar S, Powers NR, Meda SA, Calhoun VD, Gelernter J, Gruen JR, Pearlson GD (2013) Genetic influences of resting state fMRI activity in language-related brain regions in healthy controls and schizophrenia patients: a pilot study. Brain Imaging Behav 7:1527.

Jenkinson M, Smith S (2001) A global optimisation method for robust affine registration of brain images. Med Image Anal 5:143-156.

Jenkinson M, Bannister P, Brady M, Smith S (2002) Improved optimization for the robust and accurate linear registration and motion correction of brain images. Neuroimage 17:825-841.

Jenkinson M, Pechaud M, Smith S (2005) BET2: MR-based estimation of brain, skull and scalp surfaces. Eleventh annual meeting of the organization for human brain mapping, 17:167. Toronto, Canada.

Kato M, Okanoya K, Koike T, Sasaki E, Okano H, Watanabe S, Iriki A (2014) Human speech- and reading-related genes display partially overlapping expression patterns in the marmoset brain. Brain Lang 133:26-38.

Kikinis R, Pieper SD, Vosburgh KG (2014) 3D slicer: a platform for subject-specific image analysis, visualization, and clinical support. In: Intraoperative imaging and image-guided therapy, pp 277-289. Berlin: Springer Science and Business Media.

Lai CSL, Fisher SE, Hurst JA, Vargha-Khadem F, Monaco AP (2001) A forkhead-domain gene is mutated in a severe speech and language disorder. Nature 413:519-523.

Luciano M, Evans DM, Hansell NK, Medland SE, Montgomery GW, Martin NG, Wright MJ, Bates TC (2013) A genome-wide association study for reading and language abilities in two population cohorts. Genes Brain Behav 12:645-652.

Lyn HL, Pierre P, Bennett AJ, Fears SC, Woods RP, Hopkins WD (2011) Planum temporale grey matter asymmetries in chimpanzees (Pan troglodytes), vervet (Chlorocebus aethiops sabaeus), rhesus (Macaca mulatta) and bonnet (Macaca radiata) monkeys. Neuropsychologia 49:2004-2012.

Marie D, Roth M, Lacoste R, Nazarian B, Bertello A, Anton JL, Hopkins WD, Margiotoudi K, Love SA, Meguerditchian A (2018) Left brain asymmetry of the planum temporale in a nonhominid primate: redefining the origin of brain specialization for language. Cereb Cortex 28:1808-1815.

Mascheretti S, De Luca A, Trezzi V, Peruzzo D, Nordio A, Marino C, Arrigoni $F$ (2017) Neurogenetics of developmental dyslexia: from genes to behavior through brain neuroimaging and cognitive and sensorial mechanisms. Transl Psychiatry 7:e987.

Mulholland MM, Navabpour SV, Mareno MC, Schapiro SJ, Young LJ, Hopkins WD (2020) AVPR1A variation is linked to gray matter covariation in the social brain network of chimpanzees. Genes Brain Behav 19:e12631.

Muntané G, Santpere G, Verendeev A, Seeley WW, Jacobs B, Hopkins WD, Navarro A, Sherwood CC (2017) Interhemispheric gene expression differences in the cerebral cortex of humans and macaque monkeys. Brain Struct Funct 222:3241-3254.

Neal Webb SJ, Hau J, Schapiro SJ (2018) Captive chimpanzee (Pan troglodytes) behavior as a function of space per animal and enclosure type. Am J Primatol 80:e22749.

Newport EL, Hauser MD, Spaepen G, Aslin RN (2004) Learning at a distance II. Statistical learning of non-adjacent dependencies in a non-human primate. Cogn Psychol 49:85-117.
Ozernov-Palchik O, Gaab N (2016) Tackling the 'dyslexia paradox': reading brain and beahvior for early markers of developmental dyslexia. Wiley Interdiscip Rev Cogn Sci 7:156-176.

Peschansky VJ, Burbridge TJ, Volz AJ, Fiondella C, Wissner-Gross Z, Galaburda AM, Lo Turco JJ, Rosen GD (2010) The effect of variation in expression of the candidate dyslexia susceptibility gene homolog Kiaa0319 on neuronal migration and dendritic morphology in the rat. Cereb Cortex 20:884-897.

Petkov Cl, Jarvis ED (2012) Birds, primates, and spoken language origins: behavioral phenotypes and neurobiological substrates. Front Evol Neurosci 4:12.

Petkov $\mathrm{Cl}$, Kayser C, Steudel T, Whittingstall K, Augath $\mathrm{M}$, Logothetis NK (2008) A voice region in the monkey brain. Nat Neurosci 11:367-374.

Pfenning AR, Hara E, Whitney O, Rivas MV, Wang R, Roulhac PL, Howard JT, Wirthlin M, Lovell PV, Ganapathy G, Mouncastle J, Moseley MA, Thompson JW, Soderblom EJ, Iriki A, Kato M, Gilbert MTP, Zhang G, Bakken T, Bongaarts A, et al. (2014) Convergent transcriptional specializations in the brains of humans and song-learning birds. Science 346:1256846.

Pinel P, Fauchereau F, Moreno A, Barbot A, Lathrop M, Zelenika D, Le Bihan D, Poline J-B, Bourgeron T, Dehaene S (2012) Genetic variants of FOXP2 and KIAA0319/TTRAP/THEM2 locus are associated with altered brain activation in distinct language-related regions. J Neurosci 32:817-825.

Platt MP, Adler WT, Mehlhorn AJ, Johnson GC, Wright KA, Choi RT, Tsang WH, Poon MW, Yeung SY, Waye MM, Galaburda AM, Rosen GD (2013) Embryonic disruption of the candidate dyslexia susceptibility gene homolog Kiaa0319-like results in neuronal migration disorders. Neuroscience 248:585-593.

Poremba A, Saunders RC, Crane AM, Cook M, Sokoloff L, Mishkin M (2003) Functional mapping of the primate auditory system. Science 299:568-572.

Poremba A, Malloy M, Saunders RC, Carson RE, Herscovitch P, Mishkin M (2004) Species-specific calls evoke asymmetric activity in the monkey's temporal poles. Nature 427:448-451.

Prado-Martinez J, Sudmant PH, Kidd JM, Li H, Kelley JL, LorenteGaldos B, Veeramah KR, Woerner AE, O'Connor TD, Santpere G, Cagan A, Theunert C, Casals F, Laayouni H, Munch K, Hobolth A, Halager AE, Malig M, Hernandez-Rodriguez J, Hernando-Herraez I, et al. (2013) Great ape genetic diversity and population history. Nature 499:471-475.

Preuss TM (2012) Human brain evolution: from gene discovery to phenotype discovery. Proc Natl Acad Sci USA 109 [Suppl 1]:10709-10716.

Savage-Rumbaugh ES, Murphy J, Sevcik RA, Brakke KE, Williams SL, Rumbaugh DM (1993) Language comprehension in ape and child. Monogr Soc Res Child Dev 58:1-256.

Scerri TS, Morris AP, Buckingham LL, Newbury DF, Miller LL, Monaco AP, Bishop DVM, Paracchini S (2011) DCDC2, KIAA0319 and CMIP are associated with reading-related traits. Biol Psychiatry 70:237-245.

Sevcik RA, Savage-Rumbaugh ES (1994) Language comprehension and use by great apes. Lang Commun 14:37-58.

Skeide MA, Brauer J, Friederici AD (2016) Brain functional and structural predictors of language performance. Cereb Cortex 26:21272139.

Smith B, Lu CP, Alvarado Bremer J (2010) High-resolution melting analysis (HRMA): a highly sensitive inexpensive genotyping alternative for population studies. Mol Ecol Resour 10:193-196.

Smith SM (2002) Fast robust automated brain extraction. Hum Brain Mapp 17:143-155.

Smith SM, Jenkinson M, Woolrich MW, Beckmann CF, Behrens TEJ, Johansen-Berg H, Bannister PR, De Luca M, Drobnjak I, Flitney DE, Niazy RK, Saunders J, Vickers J, Zhang Y, De Stefano N, Brady JM, Matthews PM (2004) Advances in functional and structural MR image analysis and implementation as FSL. Neuroimage 23:S208-S219.

Spocter MA, Hopkins WD, Garrison AR, Bauernfeind AL, Stimpson CD, Hof PR, Sherwood CC (2010) Wernicke's area homolog in 
chimpanzees (Pan troglodytes): probabilistic mapping, asymmetry and comparison with humans. Proc Biol Sci 277:2165-2174.

Spocter MA, Hopkins WD, Barks SK, Bianchi S, Hehmeyer AE, Anderson SM, Stimpson CD, Fobbs AJ, Hof PR, Sherwood CC (2012) Neuropil distribution in the cerebral cortex differs between humans and chimpanzees. J Comp Neurol 520:2917-2929.

Sun Y, Gao Y, Zhou Y, Chen H, Wang G, Xu J, Xia J, Huen MS, Siok WT, Jiang Y, Tan LH (2014) Association study of developmental dyslexia candidate genes DCDC2 and KIAA0319 in Chinese population. Am J Med Genet B Neuropsychiatr Genet 165B:627-634.

Taglialatela JP, Russell JL, Schaeffer JA, Hopkins WD (2009) Visualizing vocal perception in the chimpanzee brain. Cereb Cortex 19:1151-1157.

Tustison N, Gee J (2009) N4ITK: Nick's N3 ITK implementation for MRI bias field correction. Insight J 9:1-8.

Tustison NJ, Avants BB, Cook PA, Zheng Y, Egan A, Yushkevich PA, Gee JC (2010) N4ITK: improved N3 bias correction. IEEE Trans Med Imaging 29:1310-1320.

Tustison NJ, Cook PA, Klein A, Song G, Das SR, Duda JT, Kandel BM, van Strien N, Stone JR, Gee JC, Avants BB (2014) Large- scale evaluation of ANTs and FreeSurfer cortical thickness measurements. Neuroimage 99:166-179.

Vargha-Khadem F, Gadian DG, Copp A, Mishkin M (2005) FOXP2 and the neuroanatomy of speech and language. Nat Rev Neurosci 6:131-138.

Vickery S, Hopkins WD, Sherwood CC, Schapiro SJ, Latzman RD, Caspers S, Gaser C, Eickhoff SB, Dahnke R, Hoffstaedter F (2020) Chimpanzee brain morphometry utilizing standardized MRI preprocessing and macroanatomical annotations. Elife 9:e60136.

Warrier C, Wong P, Penhune V, Zatorre R, Parrish T, Abrams D, Kraus N (2009) Relating structure to function: Heschl's gyrus and acoustic processing. J Neurosci 29:61-69.

Xu L, Groth KM, Pearlson G, Schretlen DJ, Calhoun VD (2009) Source-based morphometry: the use of independent component analysis to identify gray matter differences with application to schizophrenia. Hum Brain Mapp 30:711-724.

Zhu Y, Liu F, Zou X, Torbey M (2015) Comparison of unbiased estimation of neuronal number in the rat hippocampus with different staining methods. J Neurosci Methods 254:73-79. 